\title{
A Kind of Doubly Periodic Riemann Boundary Value Problem on Two Parallel Curves
}

\author{
Lixia Cao, Xiaowei Li, Chengxin Lin \\ School of Mathematics and Statistics, Northeast Petroleum University, Daqing, China \\ Email: caolixia98237@163.com
}

Received 6 November 2014; revised 3 December 2014; accepted 9 December 2014

Copyright (C) 2014 by authors and Scientific Research Publishing Inc.

This work is licensed under the Creative Commons Attribution International License (CC BY). http://creativecommons.org/licenses/by/4.0/

(c) (i) Open Access

\begin{abstract}
We proposed a kind of doubly periodic Riemann boundary value problem on two parallel curves. By using the method of complex functions, we investigated the method for solving this kind of doubly periodic Riemann boundary value problem of normal type and gave the general solutions and the solvable conditions for it.
\end{abstract}

\section{Keywords}

Normal Type, Doubly Periodic, Riemann Boundary Problem

\section{Introduction}

Various kinds of Riemann boundary value problems (BVPs) for analytic functions on closed curves or on open arcs, doubly periodic Riemann BVPs, doubly periodic or quasi-periodic Riemann BVPs and Dirichlet Problems, and BVPs for polyanalytic functions have been widely investigated in papers [1]-[8]. The main approach is to use the decomposition of polyanalytic functions and their generalization to transform the boundary value problems to their corresponding boundary value problems for analytic functions. Recently, inverse Riemann BVPs for generalized analytic functions or bianalytic functions have been investigated in papers [9]-[13].

In this paper, we consider a kind of doubly periodic Riemann boundary value problem on two parallel curves. By using the method of complex functions, we investigate the method for solving kind of doubly periodic Riemann boundary value problem of normal type and give the general solutions and the solvable conditions for it.

\section{A Kind of Doubly Periodic Riemann Boundary Value Problem on Two Parallel Curves}

Suppose that $\omega_{1}, \omega_{2}$ are complex constants with $\operatorname{Im}\left(\omega_{1} / \omega_{2}\right) \neq 0$, and $\mathbf{P}$ denotes the fundamental period pa- 
rallelogram with vertices $\pm \omega_{1} \pm \omega_{2}$. The function

$$
\zeta(z)=1 / z+\sum_{m, n} \cdot\left[1 /\left(z-\Omega_{m n}\right)+1 / \Omega_{m n}+z / \Omega_{m n}^{2}\right]
$$

is called the Weierstrass $\zeta$-function, where $\Omega_{m n}=2 m \omega_{1}+2 n \omega_{2}$, and $\sum_{m, n}{ }^{\prime}$ denotes the sum for all $m$, $n=0, \pm 1, \pm 2, \cdots$, except for $m=n=0$.

Let $L_{0}=\sum_{j=1}^{2} L_{0 j}$ be the set of two parallel curves, lying entirely in the fundamental period parallelogram $\mathbf{P}$, not passing the origin $O$, with endpoints being periodic congruent and having the same tangent lines at the periodic congruent points. Let $D_{1}, D_{2}, D_{3}$ denote the domains entirely in the fundamental period parallelogram $\mathbf{P}$, cut by $L_{01}$ and $L_{02}$, respectively. Without loss of generality, we suppose that $O \in D_{2}$, see Figure 1 . Let $L_{01}^{*}, L_{02}^{*}$ be the curves periodically extended for $L_{01}$ and $L_{02}$ with period $2 \omega_{1}$, respectively. And $L_{n j}^{*}$ $(j=1,2 ; n=0, \pm 1, \cdots)$ be the curves periodically extended for $L_{0 j}^{*}$ with $2 n \omega_{2}$.

Our objective is to find sectionally holomorphic doubly periodic functions $F(z)$ and $\Omega(z)$, satisfying the following boundary conditions

$$
\begin{cases}F^{+}(\tau)=D_{1}(\tau) \Omega^{-}(\tau)+g_{1}(\tau), & \tau \in L_{01}, \\ \Omega^{+}(\tau)=D_{2}(\tau) F^{-}(\tau)+g_{2}(\tau), & \tau \in L_{02},\end{cases}
$$

where $D_{j}(\tau), g_{j}(\tau) \in H$, and be doubly periodic with $2 \omega_{1}, 2 \omega_{2} . F^{ \pm}(\tau)$ are the boundary values of the function $F(z)$, which is analytic in $D_{1}$ and $D_{3}$, belonging to the class $h\left(a_{j}\right)$ on $L_{0 j}$, satisfying the boundary conditions (1), and $\Omega^{ \pm}(\tau)$ are the boundary values of the function $\Omega(z)$, which is analytic in $D_{2}$, belonging to the class $h\left(a_{j}\right)$ on $L_{0 j}$, satisfying the boundary conditions (1).

Since $a_{j}$ plays the same roles as other points on $L_{0 j} \quad(j=1,2)$, it is natural to require that the unknown functions are bounded at $z=a_{j}$, that is, the unknown functions $F(z)$ and $\Omega(z)$ are both bounded on $L_{01}^{*}$ and $L_{02}^{*}$.

Problem (1) is called the normal type if $D_{j}(\tau) \neq 0(j=1,2)$, otherwise the non-normal type. And if we allow the solution $\Omega(z)$ has poles of order $m$ at $z=0$, it is actually to solve problem (1) in $D R_{m}$.

\section{Preliminary Notes}

Since $D_{j}(\tau) \in H$ with $D_{j}(\tau) \neq 0(j=1,2)$, by taking logarithm of $\log D_{j}(\tau)$ for some branch on $L_{0 j}$, we may obtain a continuous single-valued function such as

$$
\begin{aligned}
& -\frac{1}{2 \pi i} \log D_{j}\left(a_{j}\right)=\alpha_{a_{j}}+i \beta_{a_{j}}, \quad j=1,2, \\
& \frac{1}{2 \pi i} \log D_{j}\left(a_{j}+2 \omega_{j}\right)=-\alpha_{a_{j}}-i \beta_{a_{j}}, \quad j=1,2 .
\end{aligned}
$$

with $0 \leq \alpha_{a_{j}}<1$. Now we call the integer $\kappa=\kappa_{1}+\kappa_{2}$ the index of problem (1), where $\kappa_{j}$ is the integer satisfying

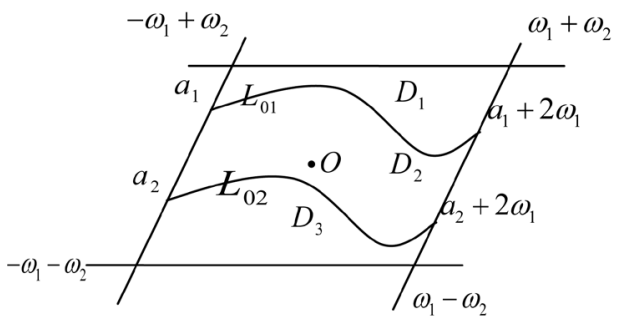

Figure 1. Parallel curves in the fundamental period parallelogram $\mathbf{P}$. 


$$
0 \leq-\alpha_{a_{j}}-\kappa_{j}<1, \quad j=1,2 .
$$

Since $\kappa_{j}$ can only be 0 and -1 , the index $\kappa$ can only take $0,-1,-2$.

Set

$$
\begin{aligned}
& D_{*}=D_{1^{*}}+D_{2^{*}}=\frac{1}{2 \pi i} \int_{L_{01}} \log D_{1}(\tau)+\frac{1}{2 \pi i} \int_{L_{02}} \log D_{2}(\tau) \mathrm{d} \tau \\
& \gamma_{j}(z)=\frac{1}{2 \pi i} \int_{L_{0 j}} \log D_{j}(\tau) \xi(\tau-z) \mathrm{d} \tau, \quad z \notin L_{0 j}, j=1,2
\end{aligned}
$$

We can easily see that $1 / \mathrm{e}^{\gamma_{j}(z)}$ will have singularities at most less than one order near the endpoints $a_{j}$ and $a_{j}+2 \omega_{1}(j=1,2)$. Let

$$
\mathrm{e}^{\gamma(z)}=\mathrm{e}^{\gamma_{1}(z)} \mathrm{e}^{\gamma_{2}(z)}
$$

then we have

$$
\mathrm{e}^{\gamma\left(z+2 \omega_{j}\right)}=\mathrm{e}^{-2 \eta_{j} D_{*}} \mathrm{e}^{\gamma(z)}, \quad j=1,2,
$$

where $\eta_{j}=\zeta\left(\omega_{j}\right)(j=1,2)$ and $2 \omega_{2} \eta_{1}-2 \omega_{1} \eta_{2}=\pi i$. Thus $\mathrm{e}^{\gamma(z)}$ is not doubly periodic generally. In fact, $\mathrm{e}^{\gamma(z)}$ is doubly periodic if and only if

$$
\eta_{j} D_{*}=k_{j} \pi i, k_{j} \text { is positive integer for } j=1,2 .
$$

Lemma 1. Formula (5) is valid if and only if

$$
\eta_{1} / \eta_{2}=k_{1} / k_{2}, \quad D_{*}=2 k_{1} \omega_{2}-2 k_{2} \omega_{1} .
$$

And if both $D_{*}=2 l_{1} \omega_{1}+2 l_{2} \omega_{2}$ and $\eta_{j} D_{*}=k_{j} \pi i$ are true, then we have $l_{1}=-k_{2}$ and $l_{2}=k_{1}$, where $l_{j}$, $k_{j}$ are all integers.

\section{Solution for Problem (1) of Normal Type}

Problem (1) can be transferred by using (3) as

$$
\begin{cases}\frac{F^{+}(\tau)}{\mathrm{e}^{\gamma_{1}^{+}}(\tau)}=\frac{\Omega^{-}(\tau)}{\mathrm{e}^{\gamma_{1}^{-}}(\tau)}+\frac{g_{1}(\tau)}{\mathrm{e}^{\gamma_{1}^{+}}(\tau)}, & \tau \in L_{01}, \\ \frac{\Omega^{+}(\tau)}{\mathrm{e}^{\gamma_{2}^{+}(\tau)}}=\frac{F^{-}(\tau)}{\mathrm{e}^{\gamma_{2}^{-}(\tau)}}+\frac{g_{2}(\tau)}{\mathrm{e}^{\gamma_{2}^{+}(\tau)}} \quad & \tau \in L_{02} .\end{cases}
$$

Multiplying $\frac{1}{\mathrm{e}^{\gamma_{2}^{-}(\tau)}}$ to the two sides of the first identity in equations (6), and multiplying $\frac{1}{\mathrm{e}^{\gamma_{1}^{-}(\tau)}}$ to the two sides of the second identity in Equations (6), gives

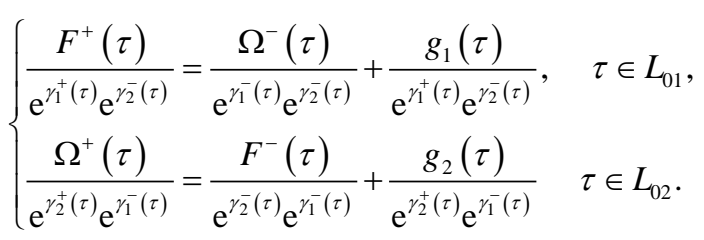

The function $1 / \mathrm{e}^{\gamma_{j}(z)}$ always has singularities at most less than one order near the endpoints $a_{j}$ and $a_{j}+2 \omega_{1}(j=1,2)$ whatever $\kappa=0,-1,-2$. And then, $\frac{g_{1}(\tau)}{\mathrm{e}^{\gamma_{1}^{+}}(\tau)} \mathrm{e}^{\gamma_{2}^{-2}(\tau)}, \frac{g_{2}(\tau)}{\mathrm{e}^{\gamma_{2}^{+}(\tau)} \mathrm{e}^{\gamma_{1}^{-}(\tau)}}$ must belong to class $H$ or class $H^{*}$ on $L_{01}$ and $L_{02}$, respectively.

Case 1. If formula (5) holds, that is, $\mathrm{e}^{\gamma(z)}$ is doubly periodic, then by Lemma 1 we have

$$
D_{*} \equiv 0\left(\bmod 2 \omega_{1}, 2 \omega_{2}\right) .
$$

Let 


$$
\begin{aligned}
& \Psi_{1}(z)=\frac{1}{2 \pi i} \int_{L_{01}} \frac{g_{1}(\tau)}{\mathrm{e}^{\gamma_{1}^{+}(\tau)} \mathrm{e}^{\gamma_{2}^{-}(\tau)}}[\zeta(\tau-z)+\zeta(z)] \mathrm{d} \tau, \quad z \notin L_{01} \\
& \Psi_{2}(z)=\frac{1}{2 \pi i} \int_{L_{02}} \frac{g_{2}(\tau)}{\mathrm{e}^{\gamma_{2}^{+}(\tau)} \mathrm{e}^{\gamma_{1}(\tau)}}[\zeta(\tau-z)+\zeta(z)] \mathrm{d} \tau, \quad z \notin L_{02}
\end{aligned}
$$

Then by formulas (9) and (10), we may rewrite (7) as

$$
\begin{cases}\frac{F^{+}(\tau)}{\mathrm{e}_{1}^{\gamma_{1}^{+}}(\tau)} \mathrm{e}^{\gamma_{2}(\tau)}-\Psi_{1}^{+}(\tau)-\Psi_{2}^{+}(\tau)=\frac{\Omega^{-}(\tau)}{\mathrm{e}^{\gamma_{1}^{-}(\tau)} \mathrm{e}^{\gamma_{2}^{-}(\tau)}}-\Psi_{1}^{-}(\tau)-\Psi_{2}^{+}(\tau), \quad \tau \in L_{01} \\ \frac{\Omega^{+}(\tau)}{\mathrm{e}^{\gamma_{2}^{+}(\tau)} \mathrm{e}^{\gamma_{1}^{-}(\tau)}}-\Psi_{1}^{-}(\tau)-\Psi_{2}^{+}(\tau)=\frac{F^{-}(\tau)}{\mathrm{e}^{\gamma_{2}^{-}(\tau)} \mathrm{e}^{\gamma_{1}^{-}(\tau)}}-\Psi_{1}^{-}(\tau)-\Psi_{2}^{-}(\tau), \quad \tau \in L_{02}\end{cases}
$$

Now we introduce the function

$$
\Phi_{1}(z)= \begin{cases}\frac{F^{+}(z)}{\mathrm{e}^{\gamma(z)}}-\Psi_{1}^{+}(z)-\Psi_{2}^{+}(z), & z \in D_{1}, \\ \frac{\Omega^{-}(z)}{\mathrm{e}^{\gamma(z)}}-\Psi_{1}^{-}(z)-\Psi_{2}^{+}(z), & z \in D_{2}, \\ \frac{F^{-}(z)}{\mathrm{e}^{\gamma(z)}}-\Psi_{1}^{-}(z)-\Psi_{2}^{-}(z), & z \in D_{3},\end{cases}
$$

then $\Phi_{1}(z)$ has $n$-order at $z=0$, and has singularities at most less than one order near the endpoints $a_{j}$ and $a_{j}+2 \omega_{1}(j=1,2)$. Thus we can get the following results.

$1^{\circ}$ When $m>0$, problem (1) is solvable without any restrictive conditions and the general solution is given by

$$
\left\{\begin{array}{l}
F^{+}(z)=\mathrm{e}^{\gamma(z)}\left[c_{0}+c_{1} \zeta^{\prime}(z)+\cdots+c_{m-1} \zeta^{m-1}(z)+\Psi_{1}^{+}(z)+\Psi_{2}^{+}(z)\right], \quad z \in D_{1}, \\
\Omega(z)=\mathrm{e}^{\gamma(z)}\left[c_{0}+c_{1} \zeta^{\prime}(z)+\cdots+c_{m-1} \zeta^{m-1}(z)+\Psi_{1}^{-}(z)+\Psi_{2}^{+}(z)\right], \quad z \in D_{2}, \\
F^{-}(z)=\mathrm{e}^{\gamma(z)}\left[c_{0}+c_{1} \zeta^{\prime}(z)+\cdots+c_{m-1} \zeta^{m-1}(z)+\Psi_{1}^{-}(z)+\Psi_{2}^{-}(z)\right], \quad z \in D_{3},
\end{array}\right.
$$

where $c_{0}, c_{1}, \cdots, c_{m-1}$ are arbitrary constants.

$2^{\circ}$ When $m=0$, problem (1) is solvable if and only if the restrictive conditions

$$
\left\{\begin{array}{l}
\frac{1}{2 \pi i} \int_{L_{01}} \frac{g_{1}(\tau)}{\mathrm{e}^{\gamma_{1}^{+}}(\tau)} \mathrm{e}^{\gamma_{2}^{-}(\tau)} \mathrm{d} \tau=0 \\
\frac{1}{2 \pi i} \int_{L_{02}} \frac{g_{2}(\tau)}{\mathrm{e}^{\gamma_{2}^{+}(\tau)} \mathrm{e}^{\gamma_{1}^{-}(\tau)}} \mathrm{d} \tau=0
\end{array}\right.
$$

are satisfied, and now the solution is given by

where $c$ is arbitrary constant.

$$
\left\{\begin{array}{l}
F^{+}(z)=\mathrm{e}^{\gamma(z)}\left[c+\Psi_{1}^{+}(z)+\Psi_{2}^{+}(z)\right], \quad z \in D_{1}, \\
\Omega(z)=\mathrm{e}^{\gamma(z)}\left[c+\Psi_{1}^{-}(z)+\Psi_{2}^{+}(z)\right], \quad z \in D_{2}, \\
F^{-}(z)=\mathrm{e}^{\gamma(z)}\left[c+\Psi_{1}^{-}(z)+\Psi_{2}^{-}(z)\right], \quad z \in D_{3},
\end{array}\right.
$$

$3^{\circ}$ When $m<0$, if and only if the restrictive conditions (13) and

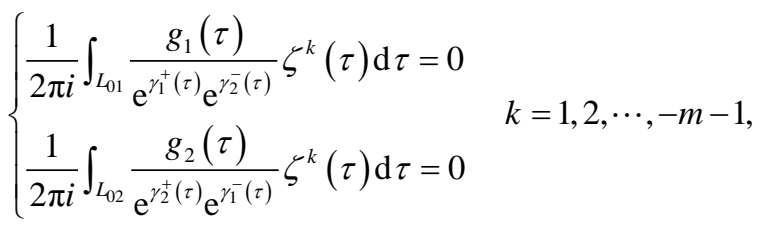


(when $m=-1$, the condition (15) is unnecessary) are necessary, problem (1) is solvable and the solution can still be given by (14) but with

$$
c=-\frac{1}{2 \pi i} \int_{L_{01}} \frac{g_{1}(\tau) \zeta^{k}(\tau)}{\mathrm{e}^{\gamma_{1}^{+}(\tau)} \mathrm{e}^{\gamma_{2}^{-}(\tau)}} \mathrm{d} \tau-\frac{1}{2 \pi i} \int_{L_{02}} \frac{g_{2}(\tau) \zeta^{k}(\tau)}{\mathrm{e}^{\gamma_{2}^{+}(\tau)} \mathrm{e}^{\gamma_{1}^{-}(\tau)}} \mathrm{d} \tau,
$$

Case 2. If formula (5) fails to hold, then by Lemma 1 we see that $D_{*} \neq 0$. Let

$$
h_{*}(z)=\sigma(z) / \sigma\left(z-D_{*}\right) \text {, }
$$

then the function $\mathrm{e}^{\gamma(z)} h_{*}(z)$ become doubly periodic, and function $\frac{1}{\mathrm{e}^{\gamma(z)} h_{*}(z)}$ has singularities at most less than one order near the endpoints $a_{j}$ and $a_{j}+2 \omega_{1}(j=1,2)$. Thus now, we can transform (6) to

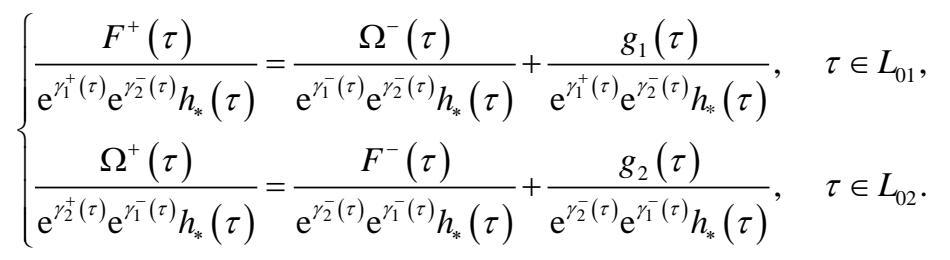

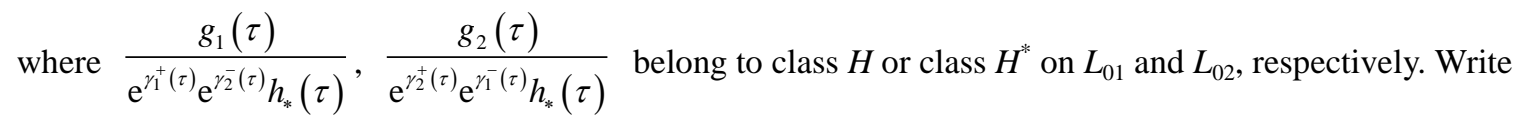

$$
\begin{aligned}
& \Psi_{* 1}(z)=\frac{1}{2 \pi i} \int_{L_{01}} \frac{g_{1}(\tau)}{\mathrm{e}^{\gamma_{1}^{+}(\tau)} \mathrm{e}^{\gamma_{2}(\tau)} h_{*}(\tau)}[\zeta(\tau-z)+\zeta(z)] \mathrm{d} \tau, \quad z \notin L_{01} \\
& \Psi_{* 2}(z)=\frac{1}{2 \pi i} \int_{L_{02}} \frac{g_{2}(\tau)}{\mathrm{e}^{\gamma_{2}^{+}(\tau)} \mathrm{e}^{\gamma_{1}^{-}(\tau)} h_{*}(\tau)}[\zeta(\tau-z)+\zeta(z)] \mathrm{d} \tau, \quad z \notin L_{02}
\end{aligned}
$$

By (17) and (18), we can rewrite (16) as

$$
\left\{\begin{array}{l}
\frac{F^{+}(\tau)}{\mathrm{e}^{\gamma_{1}^{+}(\tau)} \mathrm{e}^{\gamma_{2}^{-}(\tau)} h_{*}(\tau)}-\Psi_{* 1}^{+}(\tau)-\Psi_{* 2}^{+}(\tau)=\frac{\Omega^{-}(\tau)}{\mathrm{e}^{\gamma_{1}^{-}(\tau)} \mathrm{e}^{\gamma_{2}^{-}(\tau)} h_{*}(\tau)}-\Psi_{* 1}^{-}(\tau)-\Psi_{* 2}^{+}(\tau), \quad \tau \in L_{01}, \\
\frac{\Omega^{+}(\tau)}{\mathrm{e}^{\gamma_{2}^{+}(\tau)} \mathrm{e}^{\gamma_{1}^{-}(\tau)} h_{*}(\tau)}-\Psi_{* 1}^{-}(\tau)-\Psi_{* 2}^{+}(\tau)=\frac{F^{-}(\tau)}{\mathrm{e}^{\gamma_{2}^{-}(\tau)} \mathrm{e}^{\gamma_{1}^{-}(\tau)} h_{*}(\tau)}-\Psi_{* 1}^{-}(\tau)-\Psi_{* 2}^{-}(\tau), \quad \tau \in L_{02} .
\end{array}\right.
$$

Now we will meet two kinds of situations in solving problem (1) in $D R_{m}$.

(a) When $D_{*} \equiv 0 \bmod \left(2 \omega_{1}, 2 \omega_{2}\right)$, the function $h_{*}(z)$ is an entire function. And we can write it without counting nonzero constant as

$$
h_{*}(z)=\exp \left\{2\left(l_{1} \eta_{1}+l_{2} \eta_{2}\right)\right\}
$$

where $l_{1}, l_{2}$ are determined by the identity $D_{*}=2 l_{1} \omega_{1}+2 l_{2} \omega_{2}$.

$1^{\circ}$ When $m>0$, problem (1) is solvable without any restrictive conditions and the general solution is given by

$$
\left\{\begin{array}{l}
F^{+}(z)=\mathrm{e}^{\gamma(z)} h_{*}(z)\left[c_{0}+c_{1} \zeta^{\prime}(z)+\cdots+c_{m-1} \zeta^{m-1}(z)+\Psi_{* 1}^{+}(z)+\Psi_{* 2}^{+}(z)\right], \quad z \in D_{1}, \\
\Omega(z)=\mathrm{e}^{\gamma(z)} h_{*}(z)\left[c_{0}+c_{1} \zeta^{\prime}(z)+\cdots+c_{m-1} \zeta^{m-1}(z)+\Psi_{* 1}^{-}(z)+\Psi_{* 2}^{-}(z)\right], \quad z \in D_{2}, \\
F^{-}(z)=\mathrm{e}^{\gamma(z)} h_{*}(z)\left[c_{0}+c_{1} \zeta^{\prime}(z)+\cdots+c_{m-1} \zeta^{m-1}(z)+\Psi_{* 1}^{-}(z)+\Psi_{* 2}^{-}(z)\right], \quad z \in D_{3},
\end{array}\right.
$$

where $c_{0}, c_{1}, \cdots, c_{m-1}$ are arbitrary constants.

$2^{\circ}$ When $m=0$, problem (1) is solvable if and only if the restrictive conditions 


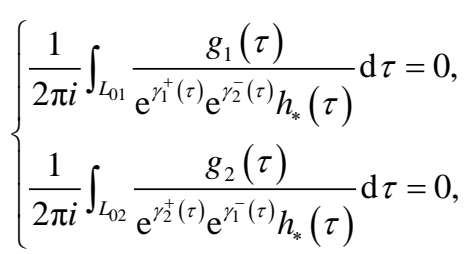

are satisfied, and the general solution for (1) is given by

$$
\begin{cases}F^{+}(z)=\mathrm{e}^{\gamma(z)} h_{*}(z)\left[c+\Psi_{* 1}^{+}(z)+\Psi_{* 2}^{+}(z)\right], & z \in D_{1}, \\ \Omega(z)=\mathrm{e}^{\gamma(z)} h_{*}(z)\left[c+\Psi_{* 1}^{-}(z)+\Psi_{* 2}^{+}(z)\right], & z \in D_{2}, \\ F^{-}(z)=\mathrm{e}^{\gamma(z)} h_{*}(z)\left[c+\Psi_{* 1}^{-}(z)+\Psi_{* 2}^{-}(z)\right], & z \in D_{3},\end{cases}
$$

where $c$ is arbitrary constant.

$3^{\circ}$ When $m<0$, if and only if the restrictive conditions (21) and

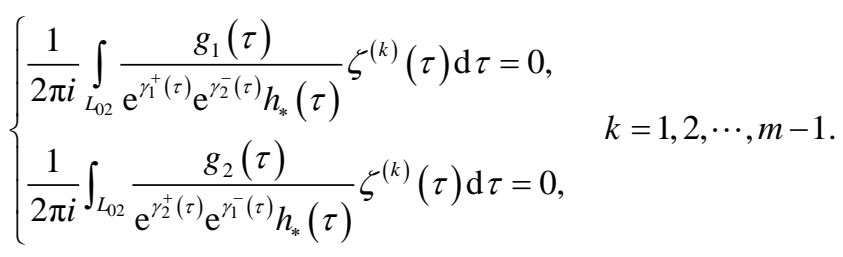

(when $m=-1$, the condition (23) is unnecessary) are both necessary, problem (1) is solvable and the solution can still be given by (22) but with

$$
c=-\frac{1}{2 \pi i} \int_{L_{01}} \frac{g_{1}(\tau)}{\mathrm{e}^{\gamma_{1}^{+}(\tau)} \mathrm{e}^{\gamma_{2}(\tau)} h_{*}(\tau)} \mathrm{d} \tau-\frac{1}{2 \pi i} \int_{L_{02}} \frac{g_{2}(\tau)}{\mathrm{e}^{\gamma_{2}^{+}(\tau)} \mathrm{e}^{\gamma_{1}(\tau)} h_{*}(\tau)} \mathrm{d} \tau .
$$

(b) When $D_{*} \equiv 0\left(\bmod 2 \omega_{1}, 2 \omega_{2}\right)$ fails to hold, the function $\frac{1}{\mathrm{e}^{\gamma(z)} h_{*}(z)}$ has singularity of one order at $z=0$, has singularities at most less than one order near the endpoints $a_{j}$ and $a_{j}+2 \omega_{1}(j=1,2)$, and has a zero of order one at $z=D_{*}$. Write

$$
\Phi_{1}(z)= \begin{cases}\frac{F^{+}(z)}{\mathrm{e}^{\gamma(z)} h_{*}(z)}-\Psi_{* 1}^{+}(z)-\Psi_{* 2}^{+}(z), & z \in D_{1}, \\ \frac{\Omega(z)}{\mathrm{e}^{\gamma(z)} h_{*}(z)}-\Psi_{* 1}^{-}(z)-\Psi_{* 2}^{+}(z), & z \in D_{2}, \\ \frac{F^{-}(z)}{\mathrm{e}^{\gamma(z)} h_{*}(z)}-\Psi_{* 1}^{-}(z)-\Psi_{* 2}^{-}(z), & z \in D_{3},\end{cases}
$$

then $\Phi_{1}(z)$ must be at most $m+1$ ordered at $z=0$, and has singularities less than one order at $z=a_{j}(j=1,2)$.

$1^{\circ}$ When $m \geq 0$, problem (1) is solvable without any restrictive conditions and the general solution is given by

$$
\left\{\begin{array}{l}
F^{+}(z)=\mathrm{e}^{\gamma(z)} h_{*}(z)\left[c_{0}+c_{1} \zeta^{\prime}(z)+\cdots+c_{m} \zeta^{m}(z)+\Psi_{* 1}^{+}(z)+\Psi_{* 2}^{+}(z)\right], \quad z \in D_{1}, \\
\Omega(z)=\mathrm{e}^{\gamma(z)} h_{*}(z)\left[c_{0}+c_{1} \zeta^{\prime}(z)+\cdots+c_{m} \zeta^{m}(z)+\Psi_{* 1}^{-}(z)+\Psi_{* 2}^{+}(z)\right], \quad z \in D_{2}, \\
F^{-}(z)=\mathrm{e}^{\gamma(z)} h_{*}(z)\left[c_{0}+c_{1} \zeta^{\prime}(z)+\cdots+c_{m} \zeta^{m}(z)+\Psi_{* 1}^{-}(z)+\Psi_{* 2}^{-}(z)\right], \quad z \in D_{3},
\end{array}\right.
$$

with the restrictive condition that

$$
c_{0}=-c_{1} \zeta^{\prime}\left(D_{*}\right)-\cdots-c_{m} \zeta^{(m)}\left(D_{*}\right)-\Psi_{* 1}\left(D_{*}\right)-\Psi_{* 2}\left(D_{*}\right)=0,
$$

or 


$$
c_{0}=-c_{1} \zeta^{\prime}\left(D_{*}\right)-\cdots-c_{m-1} \zeta^{(m)}\left(D_{*}\right)-\Psi_{* 1}\left(D_{*}\right)-\Psi_{* 2}\left(D_{*}\right),
$$

where $c_{1}, c_{2}, \cdots, c_{m}$ are arbitrary constants, which is to ensure that $\Phi_{1}\left(D_{*}\right)=0$, that is, to ensure $F^{ \pm}\left(D_{*}\right)$ and $\Omega\left(D_{*}\right)$ be bounded.

$2^{\circ}$ When $m=-1$, problem (1) is solvable if and only if the restrictive conditions

$$
\left\{\begin{array}{l}
\frac{1}{2 \pi i} \int_{L_{01}} \frac{g_{1}(\tau)}{\mathrm{e}^{\gamma_{1}^{+}(\tau)} \mathrm{e}^{\gamma_{2}(\tau)} h_{*}(\tau)} \mathrm{d} \tau=0 \\
\frac{1}{2 \pi i} \int_{L_{02}} \frac{g_{2}(\tau)}{\mathrm{e}^{\gamma_{2}^{+}(\tau)} \mathrm{e}^{\gamma_{1}^{-}(\tau)} h_{*}(\tau)} \mathrm{d} \tau=0
\end{array}\right.
$$

are satisfied, and now the solution is given by

$$
\left\{\begin{array}{l}
F^{+}(z)=\mathrm{e}^{\gamma(z)} h_{*}(z)\left[\Psi_{* 1}^{+}(z)+\Psi_{* 2}^{+}(z)-\Psi_{* 1}\left(D_{*}\right)-\Psi_{* 2}\left(D_{*}\right)\right], \quad z \in D_{1}, \\
\Omega(z)=\mathrm{e}^{\gamma(z)} h_{*}(z)\left[\Psi_{* 1}^{-}(z)+\Psi_{* 2}^{+}(z)-\Psi_{* 1}\left(D_{*}\right)-\Psi_{* 2}\left(D_{*}\right)\right], \quad z \in D_{2}, \\
F^{-}(z)=\mathrm{e}^{\gamma(z)} h_{*}(z)\left[\Psi_{* 1}^{-}(z)+\Psi_{* 2}^{-}(z)-\Psi_{* 1}\left(D_{*}\right)-\Psi_{* 2}\left(D_{*}\right)\right], \quad z \in D_{3},
\end{array}\right.
$$

which is finite at $z=D_{*}$ owing to its structure.

$3^{\circ}$ When $m<-1$, problem (1) is solvable if and only if both conditions (26) and the following conditions

$$
\begin{aligned}
& \left\{\begin{array}{l}
\frac{1}{2 \pi i} \int_{L_{01}} \frac{g_{1}(\tau)\left[\zeta(\tau)-\zeta\left(\tau-D_{*}\right)\right]}{\mathrm{e}^{\gamma_{1}^{+}(\tau)} \mathrm{e}^{\gamma_{2}^{-}(\tau)} h_{*}(\tau)} \mathrm{d} \tau=0, \\
\frac{1}{2 \pi i} \int_{L_{02}} \frac{g_{2}(\tau)\left[\zeta(\tau)-\zeta\left(\tau-D_{*}\right)\right]}{\mathrm{e}^{\gamma_{2}^{+}(\tau)} \mathrm{e}^{\gamma_{1}^{-}(\tau)} h_{*}(\tau)} \mathrm{d} \tau=0,
\end{array}\right.
\end{aligned}
$$

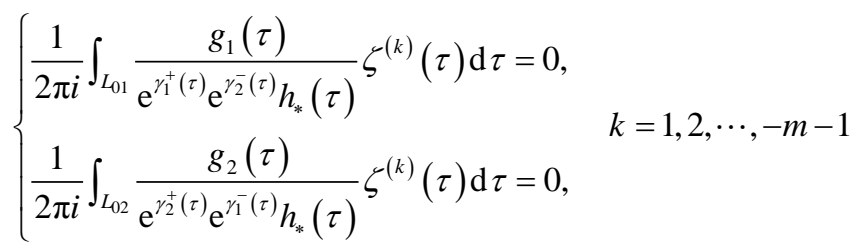

(when $m=-2$, (28) is unnecessary) are necessary, and the solution is given by

$$
\left\{\begin{array}{lc}
F^{+}(z)=\mathrm{e}^{\gamma(z)} h_{*}(z)\left[\Psi_{* 1}^{+}(z)+\Psi_{* 2}^{+}(z)-\Psi_{* 1}(0)-\Psi_{* 2}(0)\right], & z \in D_{1}, \\
\Omega(z)=\mathrm{e}^{\gamma(z)} h_{*}(z)\left[\Psi_{* 1}^{-}(z)+\Psi_{* 2}^{+}(z)-\Psi_{* 1}(0)-\Psi_{* 2}(0)\right], & z \in D_{2}, \\
F^{-}(z)=\mathrm{e}^{\gamma(z)} h_{*}(z)\left[\Psi_{* 1}^{-}(z)+\Psi_{* 2}^{-}(z)-\Psi_{* 1}(0)-\Psi_{* 2}(0)\right], & z \in D_{3} .
\end{array}\right.
$$

which is finite at $z=D_{*}$ owing to its structure.

\section{Funding}

The project of this thesis is supported by "Heilongjiang Province Education Department Natural Science Research Item", China (12541089).

\section{References}

[1] Balk, M.B. (1991) Polyanalytic Functions. Akademie Verlag, Berlin.

[2] Begehr, H. and Kumar, A. (2005) Boundary Value Problems for the Inhomogeneous Polyanalytic Equation I. Analysis: International Mathematical Journal of Analysis and its Application, 25, 55-71.

[3] Du, J.Y. and Wang, Y.F. (2003) On Boundary Value Problems of Polyanalytic Functions on the Real Axis. Complex Variables, 48, 527-542. http://dx.doi.org/10.1080/0278107031000103412 
[4] Fatulaev, B.F. (2001) The Main Haseman Type Boundary Value Problem for Metaanalytic Function in the Case of Circular Domains. Mathematical Modelling and Analysis, 6, 68-76.

[5] Lu, J.K. (1993) Boundary Value Problems for Analytic Functions. World Scientific, Singapore.

[6] Mshimba, A.S. (2002) A Mixed Boundary Value Problem for Polyanalytic Function of Order $n$ in the Sobolev Space Wn, $p(D)$. Complex Variables, 47, 278-1077.

[7] Muskhelishvili, N.I. (1993) Singular Integral Equations. World Scientific, Singapore.

[8] Wanf, Y.F. and Du, J.Y. (2006) Hilbert Boundary Value Problems of Polyanalytic Functions on the Unit Circumference. Complex Variables and Elliptic Equations, 51, 923-943. http://dx.doi.org/10.1080/17476930600667692

[9] Xing, L. (1995) A Class of Periodic Riemann Boundary Value Inverse Problems. Proceedings of the Second Asian Mathematical Conference, Nakhon Ratchasima, October 1995, 397-400.

[10] Wang, M.H. (2006) Inverse Riemann Boundary Value Problems for Generalized Analytic Functions. Journal of Ningxia University of Natural Resources and Life Sciences Education, 27, 18-24.

[11] Wen, X.Q. and Li, M.Z. (2004) A Class of Inverse Riemann Boundary Value Problems for Generalized Holomorphic Functions. Journal of Mathematical, 24, 457-464.

[12] Cao, L.X., Li, P.-R. and Sun, P. (2012) The Hilbert Boundary Value Problem With Parametric Unknown Function on Upper Half-Plane. Mathematics in Practice and Theory, 42, 189-194.

[13] Cao, L.X. (2013) Riemann Boundary Value Problem of Non-Normal Type on the Infinite Straight Line. Applied Mathematics, 4, 1126-1230. 
Scientific Research Publishing (SCIRP) is one of the largest Open Access journal publishers. It is currently publishing more than 200 open access, online, peer-reviewed journals covering a wide range of academic disciplines. SCIRP serves the worldwide academic communities and contributes to the progress and application of science with its publication.

Other selected journals from SCIRP are listed as below. Submit your manuscript to us via either submit@scirp.org or Online Submission Portal.
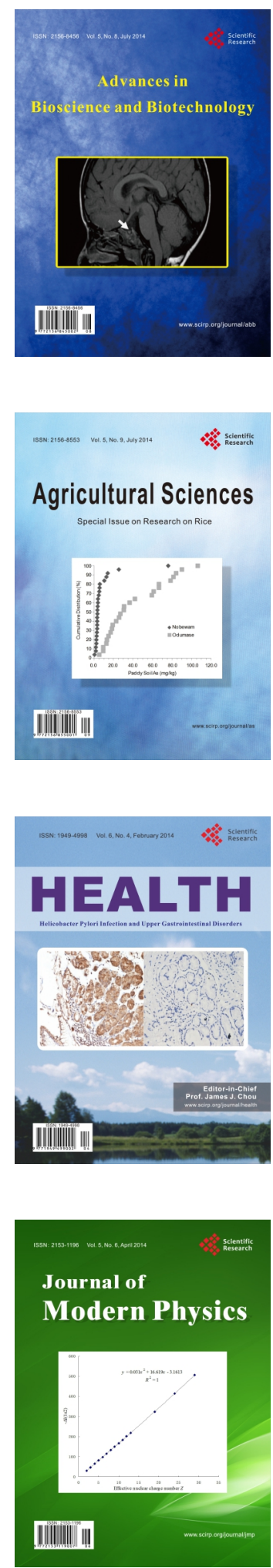
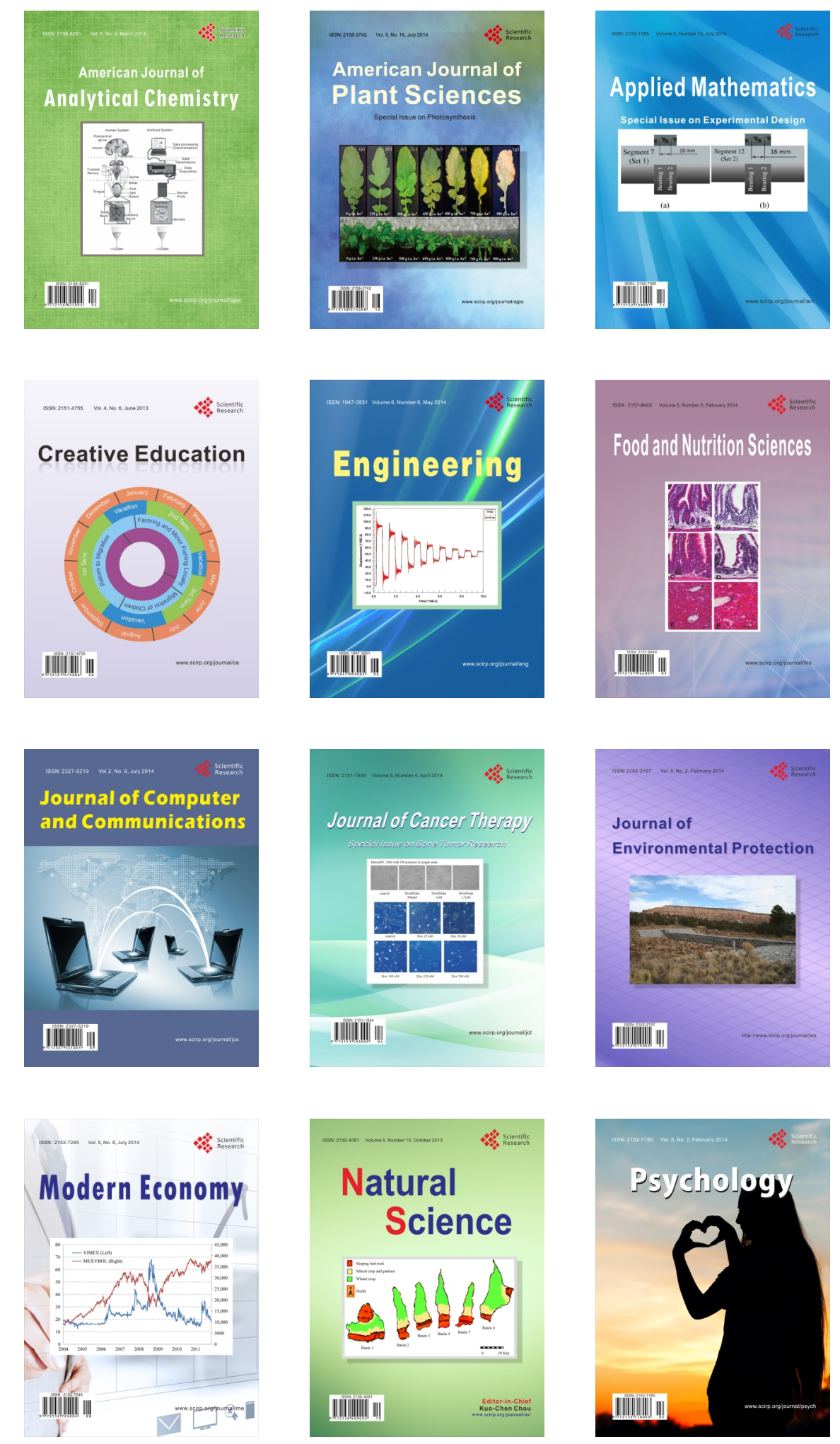